\title{
Prognostic Factors and Oncological Outcomes after Radical Nephroureterctomy for Upper Tract Urothelial Carcinoma: Review of Contemporary Multi-Center Series*
}

\author{
Ramy F. Youssef, Bishoy A. Gayed, Vitaly Margulis \\ Division of Urologic Oncology, University of Texas, Southwestern Medical Center, Dallas, USA \\ Email: ${ }^{*}$ vitaly.margulis@utsouthwestern.edu
}

Received September 11, 2012; revised October 12, 2012; accepted October 19, 2012

\begin{abstract}
Upper tract urothelial cancers (UTUC) are uncommon and the information guiding their management used to be driven from small single center studies. Multi-center international collaborations should provide clinicians with best management practices as well as prognostic factors guiding treatment decisions and outcomes. We reviewed literature from the largest multicenter collaborations for radical nephroureterctomy (RNU) performed for management of UTUC. Our review included over 50 recent manuscripts from 2009-2012 that were published from multi-center UTUC collaboration groups. Our review aims to determine of the prognostic factors predicting oncological outcomes after RNU and to provide insights about possible maximization of cure with utilization of multimodal treatment approaches. Application of approaches comprising RNU including lymphadenectomy and systemic chemotherapy; particularly in neoadjuvant settings; might have implications on improvement of oncological outcomes in high risk patients.
\end{abstract}

Keywords: Upper Tract Urothelial Carcinoma; Prognosis; Outcomes; Nephroureterctomy

\section{Introduction}

Upper tract urothelial carcinoma (UTUC) is rare disease that accounts only for $5 \%$ of UC and $7 \%-8 \%$ of renal tumors [1]. Select patients with small unifocal low grade lesions can be treated with endoscopic tumor ablation. However, Radical nephroureterectomy (RNU) with excision of an ipsilateral bladder cuff remains the gold standard treatment in patients with invasive UTUC and a functional contralateral kidney [2-4]. Despite the refinements of surgical techniques and the development of effective chemotherapeutics, oncological outcomes of patients with UTUC did not change significantly over the last decades [5]. The relative rarity of UTUC together with the heterogeneity of clinicopathological features and survival outcomes precluded prospective design of clinical studies. Clinical decision-making has historically been based on small, single center retrospective studies. These studies could not provide clinicians with a full understanding of prognostic parameters and tools to guide tailoring more effective multimodal treatment strategies.

Whereas tumor stage and histologic grade are well es-

"Conflict of Interest Statement: None of the authors of this manuscript have any financial or personal relationships to disclose that could inappropriately influence or bias our work.

${ }^{\#}$ Corresponding author. tablished prognostic factors of outcome after RNU, the prognostic significance of other potentially relevant variables, such as tumor location, architecture, necrosis, lymphovascular invasion (LVI) among others, has not been clearly established. Utilizing newly evolving prognostic factors in clinical decision making will lead to better tailoring of treatments which may potentially improve survival rates.

Defining the extent of lymph node dissection (LND) and thoughtful integration of systemic therapy may help improve treatment outcomes of patients with advanced UTUC. Neoadjuvant chemotherapy may be particularly advantageous in UTUC since the loss of renal function after RNU may render a patient ineligible for cisplatinum, the most effective agent currently in use for Urothelial cancers. Unfortunately, chemotherapy and more aggressive surgery may expose the patients to increased morbidity, and hence the need for development of selection criteria.

In this review, we sought to more clearly define management strategies, prognosis, and the impact of potential prognostic factors on oncological outcomes after surgical management of UTUC. We reviewed literature published from largest multicenter collaborations for UTUCs. We developed a library of over 50 publications from 20092012 based on huge international multi-center UTUC 
collaborations which retrospectively analyzed data from high volume European, North American or Asian centers focusing on prognostic factors and outcome of UTUC after RNU. There were two main UTUC collaborations used for the purpose of this review. The first collaboration combined data for 1462 patients who underwent RNU with ipsilateral bladder cuff resection for UTUC between 1987 and 2007 from 14 participating centers. The other collaboration aimed at validation of findings proved through the first collaboration and included 785 patients who underwent RNU between 1987 and 2008 at nine different centers, Data analyzed included clinicopathologic features and oncological outcomes. In all studies, all surgical specimens were processed according to standard pathologic procedures, and all slides were re-reviewed by genitourinary pathologists according to prospectively defined uniform criteria. All pathologists were blinded to clinical outcomes.

\section{Prognostic Factors}

All potential clinic-pathological prognostic factors were studied. Table 1 demonstrates the differences in oncological outcomes based on the most significant prognostic factors.

\section{Clinical Factors}

\subsection{Age}

The association of advanced age and poor oncological outcome for UTUC remains debatable. Elderly patients may have lower cancer specific survival (CSS) and overall survival (OS) after RNU, probably due to differences in care patterns or a decrease in the patient's defense mechanisms. In fact, utilization of LND and systemic therapy is less likely in older patients. However, many elderly patients can be cured with RNU suggesting that advanced age alone should not hinder aggressive management of potentially curable UTUC [6,7]. Performance status should be combined with age in calculation of treatment options for elderly cancer patients.

\subsection{Gender}

Gender does not seem to affect the biological behavior of UTUC or outcomes after RNU. In a large multicenter study, gender was not among the independent predictors of oncological outcomes after RNU as shown in univariate and multivariate Cox regression analyses predicting disease recurrence and cancer-specific mortality [8].

\subsection{Obesity}

Obesity adversely impacts oncological outcomes in patients with UTUC (Table 1). Ehdaie et al., found 37\%
Table 1. 3Y and 5Y survival rates based on different clinicpathological parameters.

\begin{tabular}{|c|c|c|c|c|}
\hline & \multicolumn{2}{|c|}{ DFS (\%) } & \multicolumn{2}{|c|}{$\operatorname{CSS}(\%)$} \\
\hline & $3 Y$ & $5 Y$ & $3 Y$ & $5 Y$ \\
\hline \multicolumn{5}{|l|}{ Stage } \\
\hline $\mathrm{T}_{0}, \mathrm{Ta}$, Tis & 94 & 92 & 97 & 94 \\
\hline $\mathrm{T} 1$ & 89 & 88 & 92 & 91 \\
\hline $\mathrm{T} 2$ & 75 & 71 & 81 & 75 \\
\hline $\mathrm{T} 3$ & 52 & 48 & 62 & 54 \\
\hline $\mathrm{T} 4$ & 16 & 5 & 24 & 12 \\
\hline \multicolumn{5}{|l|}{ Grade } \\
\hline Low & 92 & 88 & 94 & 89 \\
\hline High & 60 & 57 & 69 & 63 \\
\hline \multicolumn{5}{|l|}{$\mathbf{L N}$} \\
\hline No & 77 & 73 & 92 & 77 \\
\hline Yes & 30 & 23 & 41 & 35 \\
\hline \multicolumn{5}{|l|}{ LVI } \\
\hline No & 80 & 77 & 85 & 80 \\
\hline Yes & 48 & 44 & 58 & 50 \\
\hline \multicolumn{5}{|l|}{ Architecture } \\
\hline Papillary & 81 & 79 & 86 & 82 \\
\hline Sessile & 45 & 40 & 55 & 46 \\
\hline \multicolumn{5}{|l|}{ Concomitant CIS } \\
\hline No & 77 & 74 & 81 & 76 \\
\hline Yes & 56 & 56 & 66 & 60 \\
\hline \multicolumn{5}{|l|}{ BMI } \\
\hline$<25$ & 86 & 86 & 86 & 84 \\
\hline $25-29.9$ & 84 & 74 & 70 & 65 \\
\hline$\geq 30$ & 59 & 49 & 53 & 47 \\
\hline \multicolumn{5}{|l|}{ Symptoms } \\
\hline Asymptomatic & 77 & 73 & 79 & 76 \\
\hline Local symptoms & 70 & 69 & 74 & 72 \\
\hline Systemic symptoms & 39 & 34 & 40 & 35 \\
\hline
\end{tabular}

difference in both $5 \mathrm{Y}$-disease free survival (DFS) and 5 Y-CSS rates between patients with BMI $\geq 30(49 \%$, $47 \%)$ compared to patients with BMI $<25(86 \%, 84 \%)$ [9].

\subsection{Preopertaive Symptoms}

The presence of symptoms as well as the performance status is well known prognostic factors in kidney cancer. Multicenter UTUC collaboration could not find a significant difference in oncological outcomes between asymptomatic compared to patients with local symptoms [10]. However, The presence of systemic symptoms such as weight loss, anorexia and bone pain were usually associated with worse oncological outcomes, even in patients suspected to have clinically localized disease [10]. One could assume that, patients with systemic symptoms may harbor micrometastatic disease and could potentially benefit from a more rigorous metastatic evaluation or perioperative chemotherapy regimens. 


\subsection{Hydronephrosis}

Hydronephrosis was shown to be a sign of advanced disease and poor outcome in both bladder cancer and UTUC [11-13]. Brien et al., found hydronephrosis, positive cytology and high ureteroscopic grade to be independently associated with non-organ confined (NOC-UTUC). Abnormality of all three variables had $89 \%$ and $73 \%$ positive predictive value for $\mathrm{pT}_{2}+$ and NOC-UTUC, respectively, but when all tests were normal, the negative predictive value was $100 \%$ [14]. Preoperative evaluation for hydronephrosis can identify patients at risk of non-organconfined UTUC and such knowledge might impact treatment choice, including consideration of perioperative chemotherapy [14].

\section{Pathological Factors}

\subsection{Tumor Stage}

Pathological stage remains the most important predictor of DFS and CSS in UTUC. Table 1 demonstrates the differences in survival rates for different pathological $\mathrm{T}$ stages. The metastatic potential increases with advancing tumor stage and therefore prognosis worsens and becomes dismal in $\mathrm{T}_{4}$ disease which has a $5 \mathrm{Y}$-DFS of less than $5 \%$. The risk of disease recurrence or death due to cancer was almost doubled with every step higher at pathological $\mathrm{T}$ stage [3]. Integration of perioperative chemotherapy, particularly in neoadjuvant settings with aggressive RNU including LND might be the only available treatment option that might improve the dismal prognosis of locally advanced UTUC.

\subsection{Tumor Grade}

Grade is not only an independent predictor of oncological outcome but also one of the most important parameters for decision making in treatment of UTUC. Unsurprisingly, $2 / 3$ of the patients has high grade disease at RNU [3]. We were able to create a nomogram which can accurately predict non-organ confined UTUC preoperatively based on three readily available parameters; grade, architecture and location of the tumor. Tumor grade represented the most influential predictor and spans the entire spectrum of risk points $(0-100$ points $)$ in this nomogram followed by architecture then, lastly, tumor location [15]. This simple preoperative prediction model can be utilized for patient counseling, selection for neoadjuvant systemic therapy and guidance to the extent of LND during RNU.

\subsection{Lympadenectomy, LN Status and Nodal Prognostic Factors}

Nodal status is one of the important predictors of DFS and CSS in UTUC [3,16-19]. Roscigno et al., found pNx to be significantly associated with a worse prognosis than $\mathrm{pN}_{0}$ in $\mathrm{pT}_{2-4}$ tumors [17-18,20]. LND might have more significant impact on outcome in patients expected to have $\mathrm{pT}_{2-4}$ disease, while it might not make a survival difference in those with localized organ confined disease. The probability of LN involvement increases in patients with higher $\mathrm{T}$ stages, for whom, LND might improve staging and thereby help guide decision making regarding adjuvant chemotherapy [17,21-22]. A minimum of 8 removed LNs seemed to be the most informative cutoff with $75 \%$ probability of finding 1 positive node [18]. Meanwhile, 30\% LN density (defined as the number of positive nodes divided by the total number of LNs removed) seems to be the most prognostic [23]. Recently, extranodal extension was found to be a powerful prognostic factor [24].

LNs were found to be involved in $20 \%$ - $25 \%$ of patients who underwent LND during RNU [3,17,18,20]. With the biological similarity and from the model of UCB, extended LND during RNU may provide with more accurate staging and might be curative for patients with limited nodal involvement, particularly in the era of neoadjuvant chemotherapy. However, the indications and extent of LND during RNU are still not standardized, since the current evidence is based on retrospective studies and prospective trials are still needed [16]. Moreover, Even in academic centers, LND was performed only in $40 \%-50 \%$ of cases [16].

\subsection{Lymphovascular Invasion}

Lymphatic vessels serve as the primary pathway for metastatic tumor cell spread, and so lymphovascular invasion (LVI) has an important prognostic role in most malignancies, including UTUC. LVI was recently reported in $\approx 25 \%$ of RNU specimens and was associated with established features of biologically aggressive UTUC, such as advanced stage, high tumor grade, LN metastasis, sessile tumor architecture, tumor necrosis, and concomitant carcinoma in situ. Moreover, LVI has been identified as an independent predictor of disease recurrence and cancer-specific mortality $[3,25,26]$. Addition of LVI to standard pathologic features (pathologic $\mathrm{T}$ stage, grade, and LN status) has been demonstrated to improve predictive accuracy for both disease recurrence and cancerspecific mortality by a statistically significant, but clinically small, margin. This margin was larger when the analyses were restricted to patients without LN metastasis and those who did not undergo LND [25].

\subsection{Tumor Architecture}

Sessile tumor architecture was reported in $\approx 25 \%-30 \%$ of UTUC surgically removed by RNU [27,28]. Finding 
sessile rather than a papillary architecture, was associated with established features of biologically aggressive UTUC, such as advanced stage, high tumor grade and LN metastasis. It has been shown to independently predict oncological outcomes after RNU [3,27,28]. Table 1 shows the difference in survival rates of patients with papillary cancers and those with sessile tumors. The previously mentioned preoperative nomogram combining tumor architecture with histological grade and tumor location achieved $76.6 \%$ accuracy for predicting nonorgan-confined UTUC [15].

\subsection{Carcinoma in Situ}

Carcinoma in situ (CIS) is a flat intraepithelial lesion characterized by marked cytologic abnormalities, and is considered as a marker of genetic instability associated with development of multifocal metachronus tumors [29]. In UTUC, CIS has been demonstrated to be a significant independent predictor of subsequent bladder recurrence. Furthermore, presence of concomitant CIS in patients with organ-confined UTUC is associated with aggressive pathological features and worse oncological outcomes after RNU [30,31]. Moreover, history of bladder CIS was associated with higher risk of recurrence and death from cancer after RNU (5Y DFS and CSS were 53\%, 59\% versus $71 \%$ and $75 \%$ in those without history of bladder CIS), suggesting the need for aggressive surveillance regimens and multimodal management strategies in patients who develop UTUC after a history of bladder CIS [29].

\subsection{Necrosis}

Extensive tumor necrosis (defined as $>10 \%$ of the tumor area) was reported in $22 \%$ of UTUC during pathological evaluation of RNU specimens and was associated with other aggressive pathological features including high grade, LN metastasis, LVI, sessile architecture and concomitant CIS. Moreover, it was also an independent predictor of oncological outcomes after RNU in UTUCc [32]. However, this was not validated in UTUCv, as it was only associated with a worse outcome on univariate analysis; but could not maintain independent prognostic value in multivariate analysis [33]. Further validation studies are needed before tumor necrosis can be used to guide clinical decision-making after RNU.

\subsection{Tumor Location}

It is still debatable whether tumor location has a significant impact on outcomes of UTUC. The difference in oncological outcomes between tumors in the pelvicalyceal system versus ureteric tumors might be not significant [34]. However, studying patients that were treated with RNU after development of tumors at ureteroenteric junction after urinary diversion, we could identify the aggressive behavior of these peculiar tumors and the poor outcomes, which calls for use of multimodal treatment including systemic chemotherapy in treatment of these aggressive malignancies [35].

\section{Management}

\subsection{Surgical Management}

RNU is still the gold standard treatment of invasive and/or high grade UTUC. Outcomes after RNU did not change significantly over the time, despite staging and surgical refinements and availability of active systemic treatment. Minimally invasive surgical modalities for UTUC management (endoscopic tumor ablation or Laparoscopic RNU) are more utilized for selected patients. Oncological outcomes after laparoscopic RNU were comparable to open RNU. However, laparoscopic RNU is usually selectively performed in favorable-risk patients [36-38]. Previous history of endoscopic tumor ablation may not compromise oncological outcomes after RNU, alleviating the concerns about the oncological safety of endoscipc ablation and possible progression with delayed RNU [4].

\subsection{Perioperative Chemotherapy}

Utilization of peri-operative systemic chemotherapy in UTUC management remains low $[15,39,40]$. Improvements in treatment outcomes necessitate rigorous investigation and application of multi-modal treatment approaches and enhanced identification of high-risk patients.

\subsection{Neoadjuvant Chemotherapy}

Despite the compelling evidence for the use of neoadjuvant platinum-based chemotherapy for high risk UCB, few patients receive neoadjuvant chemotherapy prior to RNU. The potential advantages of neoadjuvant chemotherapy in UTUC include potential eradication of subclinical metastases, improved patient tolerability prior to surgical extirpation and ability to deliver higher chemotherapy doses prior to loss of global renal function. Studying advanced UTUC cases with loco-regional nodal metastases, we found that neoadjuvant systemic therapy followed by aggressive surgical consolidation may improve oncologic outcomes. This approach represents a promising treatment strategy for UTUC patients with known or at risk for advanced disease [39]. A similar finding was found in $\mathrm{T}_{4}$ tumors (unpublished data).

\subsection{Adjuvant Chemotherapy}

There is an overall higher utilization of adjuvant compared to neoadjuvant chemotherapy in management of 
UTUC [40]. However, adjuvant chemotherapy is still infrequently used to treat high risk UTUC after RNU (in a large multicenter collaboration including 1390 patients with $542(39 \%)$ high risk UTUC $\left(\mathrm{pT}_{3} \mathrm{~N}_{0}, \mathrm{pT}_{4} \mathrm{~N}_{0}\right.$ and/or lymph node positive), only $12 \%$ of all and $22 \%$ of high risk UTUC patients, received adjuvant chemotherapy). Adjuvant chemotherapy had minimal impact on oncological outcomes. In fact, adjuvant chemotherapy imparted a trend toward slightly worse CSS and OS, largely because it was administered more often in patients with higher stage and grade [40].

\subsection{Future UTUC Collaborative Research Exploring Molecular Biomarkers}

Future research into prognostic biomarkers for UTUC and integration with other clinic-pathological parameters may guide selection for systemic therapies and tailoring of individualized multimodal treatments as well as design of clinical trials. This might potentially improve the management and outcomes of UTUC patients. We started to explore multiple biomarkers involved in different cancer pathways and we found a panel of cell cycle related biomarkers to be promising [41]. Incorporation of biomarkers into clinical practice might potentially allow an enhanced patient counseling, individualized (neo)adjuvant chemotherapy recommendations, and patient-specific surveillance regimens.

\section{Conclusion}

Despite the refinement of surgical modalities, there was no significant improvement in oncological outcomes after management of UTUC, reflecting the underutilization of multimodal treatment approaches. Understanding the biology of the disease and accurate stratification of patients based on emerging prognostic indicators may enable tailoring more effective multimodal treatments and design of clinical trials. Pathological stage, grade, LVI, tumor architecture are the most important pathological prognostics. Systemic therapy, particularly neoadjuvant chemotherapy, combined with aggressive surgical consolidation might improve oncologic outcomes in high risk UTUC patients.

\section{REFERENCES}

[1] A. Jemal, et al., "Cancer Statistics, 2010," A Cancer Journal for Clinicians, Vol. 60, No. 5, 2010, pp. 277-300. doi:10.3322/caac.20073

[2] M. Roupret, et al., "European Guidelines for the Diagnosis and Management of Upper Urinary Tract Urothelial Cell Carcinomas: 2011 Update," European Urology, Vol. 59, No. 4, 2011, pp. 584-594.

[3] V. Margulis, et al., "Outcomes of Radical Nephroureterectomy: A Series from the Upper Tract Urothelial Carci- noma Collaboration," Cancer, Vol. 115, No. 6, 2009, pp. 1224-1233. doi:10.1002/cncr.24135

[4] C. Gurbuz, et al., "The Impact of Previous Ureteroscopic Tumor Ablation on Oncologic Outcomes after Radical Nephrouretectomy for Upper Urinary Tract Urothelial Carcinoma," Journal of Endourology, Vol. 25, No. 5, 2011, pp. 775-779. doi:10.1089/end.2010.0396

[5] M. Adibi, et al., "Oncological Outcomes after Radical Nephroureterectomy for Upper Tract Urothelial Carcinoma: Comparison over the Three Decades," International Journal of Urology, 2012.

[6] S. F. Shariat, et al., "Advanced Patient Age Is Associated with Inferior Cancer-Specific Survival after Radical Nephroureterectomy," BJU International, Vol. 105, No. 12, 2010, pp. 1672-1677

doi:10.1111/j.1464-410X.2009.09072.x

[7] T. F. Chromecki, et al., "Chronological Age Is Not an Independent Predictor of Clinical Outcomes after Radical Nephroureterectomy," World Journal of Urology, Vol. 29, No. 4, 2011, pp. 473-480. doi:10.1007/s00345-011-0677-0

[8] M. I. Fernandez, et al., "Evidence-Based Sex-Related Outcomes after Radical Nephroureterectomy for Upper Tract Urothelial Carcinoma: Results of Large Multicenter Study," Urology, Vol. 73, No. 1, 2009, pp. 142-146. doi:10.1016/j.urology.2008.07.042

[9] B. Ehdaie, et al., "Obesity Adversely Impacts Disease Specific Outcomes in Patients with Upper Tract Urothelial Carcinoma," Journal of Urology, Vol. 186, No. 1, 2011, pp. 66-72. doi:10.1016/j.juro.2011.03.031

[10] J. D. Raman, et al., "Does Preoperative Symptom Classification Impact Prognosis in Patients with Clinically Localized Upper-Tract Urothelial Carcinoma Managed by Radical Nephroureterectomy?" Urologic Oncology, Vol. 29, No. 6, 2010, pp. 716-723.

[11] C. K. Ng, et al., "Does the Presence of Hydronephrosis on Preoperative Axial CT Imaging Predict Worse Outcomes for Patients Undergoing Nephroureterectomy for Upper-Tract Urothelial Carcinoma?" Urologic Oncology, Vol. 29, No. 1, 2011, pp. 27-32. doi:10.1016/j.urolonc.2008.10.023

[12] K. S. Cho, et al., "Grade of Hydronephrosis and Tumor Diameter as Preoperative Prognostic Factors in Ureteral Transitional Cell Carcinoma," Urology, Vol. 70, No. 4, 2007, pp. 662-666. doi:10.1016/j.urology.2007.06.1106

[13] C. J. Stimson, et al., "Preoperative Hydronephrosis Predicts Extravesical and Node Positive Disease in Patients Undergoing Cystectomy for Bladder Cancer," Journal of Urology, Vol. 183, No. 5, 2010, pp. 1732-1737. doi:10.1016/j.juro.2010.01.028

[14] J. C. Brien, et al., "Preoperative Hydronephrosis, Ureteroscopic Biopsy Grade and Urinary Cytology Can Improve Prediction of Advanced Upper Tract Urothelial Carcinoma," Journal of Urology, Vol. 184, No. 1, 2010, pp. 69-73. doi:10.1016/j.juro.2010.03.030

[15] V. Margulis, et al., "Preoperative Multivariable Prognostic Model for Prediction of Nonorgan Confined Urothelial Carcinoma of the Upper Urinary Tract," Journal of Urology, Vol. 184, No. 2, 2010, pp. 453-458. 
doi:10.1016/j.juro.2010.03.142

[16] M. Roscigno, et al., "Lymphadenectomy at the Time of Nephroureterectomy for Upper Tract Urothelial Cancer," European Urology, Vol. 60, No. 4, 2011, pp. 776-783.

[17] M. Roscigno, et al., "Impact of Lymph Node Dissection on Cancer Specific Survival in Patients with Upper Tract Urothelial Carcinoma Treated with Radical Nephroureterectomy," Journal of Urology, Vol. 181, No. 6, 2009, pp. 2482-2489. doi:10.1016/j.juro.2009.02.021

[18] M. Roscigno, et al., "The Extent of Lymphadenectomy Seems to Be Associated with Better Survival in Patients with Nonmetastatic Upper-Tract Urothelial Carcinoma: How Many Lymph Nodes Should Be Removed?" European Urology, Vol. 56, No. 3, 2009, pp. 512-518. doi:10.1016/j.eururo.2009.06.004

[19] R. J. Mason, et al., "The Contemporary Role of Lymph Node Dissection during Nephroureterectomy in the Management of Upper Urinary Tract Urothelial Carcinoma: The Canadian Experience," Urology, Vol. 79, No. 4, 2012, pp. 840-845. doi:10.1016/j.urology.2011.11.058

[20] M. Roscigno, et al., "Assessment of the Minimum Number of Lymph Nodes Needed to Detect Lymph Node Invasion at Radical Nephroureterectomy in Patients with Upper Tract Urothelial Cancer," Urology, Vol. 74, No. 5, 2009, pp. 1070-1074. doi:10.1016/j.urology.2009.04.084

[21] M. Burger, et al., "No Overt Influence of Lymphadenectomy on Cancer-Specific Survival in Organ-Confined Versus Locally Advanced Upper Urinary Tract Urothelial Carcinoma Undergoing Radical Nephroureterectomy: A Retrospective International, Multi-Institutional Study," World Journal of Urology, Vol. 29, No. 4, 2011, pp. 465-472. doi:10.1007/s00345-011-0705-0

[22] G. Lughezzani, et al., "A Critical Appraisal of the Value of Lymph Node Dissection at Nephroureterectomy for Upper Tract Urothelial Carcinoma," Urology, Vol. 75, No. 1, 2010, pp. 118-124. doi:10.1016/j.urology.2009.07.1296

[23] C. Bolenz, et al., "Risk Stratification of Patients with Nodal Involvement in Upper Tract Urothelial Carcinoma: Value of Lymph-Node Density," BJU International, Vol. 103, No. 3, 2009, pp. 302-306. doi:10.1111/j.1464-410X.2008.07988.x

[24] H. Fajkovic, et al., "Prognostic Value of Extranodal Extension and Other Lymph Node Parameters in Patients with Upper Tract Urothelial Carcinoma," Journal of Urology, Vol. 187, No. 3, 2012, pp. 845-851. doi:10.1016/j.juro.2011.10.158

[25] E. Kikuchi, et al., "Lymphovascular Invasion Predicts Clinical Outcomes in Patients with Node-Negative Upper Tract Urothelial Carcinoma," Journal of Clinical Oncology, Vol. 27, No. 4, 2009, pp. 612-618. doi:10.1200/JCO.2008.17.2361

[26] G. Novara, et al., "Prognostic Role of Lymphovascular Invasion in Patients with Urothelial Carcinoma of the Upper Urinary Tract: An International Validation Study," European Urology, Vol. 57, No. 6, 2010, pp. 1064-1071. doi:10.1016/j.eururo.2009.12.029

[27] H. M. Fritsche, et al., "Macroscopic Sessile Tumor Architecture Is a Pathologic Feature of Biologically Aggres- sive Upper Tract Urothelial Carcinoma," Urologic Oncology, Vol. 30, No. 5, 2010, pp. 666-672.

[28] M. Remzi, et al., "Tumour Architecture Is an Independent Predictor of Outcomes after Nephroureterectomy: A Multi-Institutional Analysis of 1363 Patients," BJU International, Vol. 103, No. 3, 2009, pp. 307-311. doi:10.1111/j.1464-410X.2008.08003.X

[29] R. F. Youssef, et al., "Prognostic Effect of Urinary Bladder Carcinoma in Situ on Clinical Outcome of Subsequent Upper Tract Urothelial Carcinoma," Urology, Vol. 77, No. 4, 2011, pp. 861-866. doi:10.1016/j.urology.2010.09.032

[30] J. C. Wheat, et al., "Concomitant Carcinoma in Situ Is a Feature of Aggressive Disease in Patients with Organ Confined Urothelial Carcinoma Following Radical Nephroureterectomy," Urologic Oncology, Vol. 30, No. 3, 2012, pp. 252-258. doi:10.1016/j.urolonc.2010.01.001

[31] W. Otto, et al., "Concomitant Carcinoma in Situ as an Independent Prognostic Parameter for Recurrence and Survival in Upper Tract Urothelial Carcinoma: A Multicenter Analysis of 772 Patients," World Journal of Urology, Vol. 29, No. 4, 2011, pp. 487-494. doi:10.1007/s00345-011-0645-8

[32] R. Zigeuner, et al., "Tumour Necrosis Is an Indicator of Aggressive Biology in Patients with Urothelial Carcinoma of the Upper Urinary Tract," European Urology, Vol. 57, No. 4, 2010, pp. 575-581. doi:10.1016/j.eururo.2009.11.035

[33] C. Seitz, et al., "Association of Tumor Necrosis with Pathological Features and Clinical Outcome in $754 \mathrm{~Pa}-$ tients Undergoing Radical Nephroureterectomy for Upper Tract Urothelial Carcinoma: An International Validation Study," Journal of Urology, Vol. 184, No. 5, 2010, pp. 1895-1900. doi:10.1016/j.juro.2010.06.106

[34] J. D. Raman, et al., "Impact of Tumor Location on Prognosis for Patients with Upper Tract Urothelial Carcinoma Managed by Radical Nephroureterectomy," European Urology, Vol. 57, No. 6, 2010, pp. 1072-1079. doi:10.1016/j.eururo.2009.07.002

[35] R. F. Youssef, et al., "Urothelial Carcinoma at the Uretero-Enteric Junction: Multi-Center Evaluation of Oncologic Outcomes after Radical Nephroureterectomy," Urologic Oncology, 2011. http://dx.doi.org/10.1016/j.urolonc.2011.05.006

[36] U. Capitanio, et al., "Comparison of Oncologic Outcomes for Open and Laparoscopic Nephroureterectomy: A Multi-Institutional Analysis of 1249 Cases," European Urology, Vol. 56, No. 1, 2009, pp. 1-9. doi:10.1016/j.eururo.2009.03.072

[37] T. J. Walton, et al., "Oncological Outcomes after Laparoscopic and Open Radical Nephroureterectomy: Results from an International Cohort," BJU International, Vol. 108, No. 3, 2011, pp. 406-412. doi:10.1111/j.1464-410X.2010.09826.x

[38] M. M. Ariane, et al., "Assessment of Oncologic Control Obtained after Open versus Laparoscopic Nephroureterectomy for Upper Urinary Tract Urothelial Carcinomas (UUT-UCs): Results from a Large French Multicenter Collaborative Study," Annals of Surgical Oncology, Vol. 
19, No. 1, 2012, pp. 301-308. doi:10.1245/s10434-011-1841-x

[39] R. F. Youssef, et al., "Upper Urinary Tract Urothelial Carcinoma with Loco-Regional Nodal Metastases: Insights from the Upper Tract Urothelial Carcinoma Collaboration," BJU International, Vol. 108, No. 8, 2011, pp. 1286-1291.

[40] N. J. Hellenthal, et al., "Adjuvant Chemotherapy for High Risk Upper Tract Urothelial Carcinoma: Results from the
Upper Tract Urothelial Carcinoma Collaboration," Journal of Urology, Vol. 182, No. 3, 2009, pp. 900-906. doi:10.1016/j.juro.2009.05.011

[41] A. Bagrodia, et al., "Prospective Evaluation of Molecular Markers for the Staging and Prognosis of Upper Tract Urothelial Carcinoma," European Urology, Vol. 62, No. 1, 2012, pp. 27-29. doi:10.1016/j.eururo.2012.04.031 